\title{
Lobar cerebral microbleeds signal early cognitive impairment
}

Yusuke Yakushiji ${ }^{1} \&$ David J. Werring ${ }^{2}$

${ }^{1}$ Division of Neurology, Department of Internal Medicine, Saga University Faculty of Medicine, 5-1-1 Nabeshima, Saga, 849-8501, Japan

${ }^{2}$ Stroke Research Centre, Department of Brain Repair \& Rehabilitation, UCL Institute of Neurology and The National Hospital for Neurology and Neurosurgery, Queen Square, London, UK, WC1N 3BG

\section{Corresponding author}

Correspondence to: Yusuke Yakushiji, Division of Neurology, Department of Internal Medicine, Saga University Faculty of Medicine, 5-1-1 Nabeshima, Saga, 849-8501, Japan; Phone: +81-95234-2360, Fax: +81-952-34-2017; E-mail: yakushij@cc.saga-u.ac.jp

\section{Author biographies (approximately 100 words)}

Yusuke Yakushiji is Lecturer of Neurology and Vice-Director of the Cerebrovascular Center at Saga University, Saga, Japan. He studies the association between cerebral small vessel disease and neurocognitive disorders, and its ethnic or geographical differences, often in collaboration with David J. Werring

David J. Werring is Professor of Clinical Neurology at the Stroke Research Centre, University College London Institute of Neurology, London, UK. He is also a consultant neurologist at the National Hospital for Neurology and Neurosurgery and University College Hospital, London, UK, where he contributes to the delivery of hyperacute, acute and outpatient stroke care, and runs a specialist clinical service and research program in intracerebral haemorrhage and small vessel disease. 
Yakushiji Y \& Werring D.J.

A cross-sectional community-based study shows that strictly lobar cerebral microbleeds are associated with cognitive impairment, and might be a surrogate marker for early smallvessel injury. The anatomical distribution of cerebral microbleeds suggests that cerebral amyloid angiopathy (with or without Alzheimer disease) might play a key role in early cognitive impairment.

Refers to Chung CP. et al. Strictly lobar cerebral microbleeds are associated with cognitive impairment.

Stroke. http://stroke.ahajournals.org/content/early/2016/09/13/STROKEAHA.116.014166 
Cerebral microbleeds (CMBs) have been associated with cognitive impairment, but the nature of the relationship and the mechanisms involved are unclear. In this context, the locations of CMBs are of great interest, because they are likely to indicate underlying small vessel pathology that underlies the cognitive dysfunction. A community-based Taiwanese population study by Chung et al., recently published in Stroke ${ }^{1}$, adds important new data. The study shows that strictly lobar CMBs are associated with cognitive impairment, providing further insight into the likely pathogenic mechanisms, and, specifically, suggesting that cerebral amyloid angiopathy (CAA) and Alzheimer disease (AD) might be important contributory factors.

In the late 1990s, advances in MRI techniques allowed in vivo detection of very small foci of chronic blood products in the brains of patients with cerebral small vessel disease (SVD). These lesions, now commonly referred to as cerebral microbleeds $(\mathrm{CMBs})^{2}$, cause strong paramagnetic distortion of the local magnetic field (so-called blooming), making them conspicuous on $\mathrm{T} 2 *$-weighted gradient-recalled echo sequences or susceptibility-weighted MRI sequences $^{2}$; they appear as small $(<10 \mathrm{~mm}$ diameter $)$, rounded, homogeneous, hypointense lesions. Usually, CMBs correspond pathologically to near-microscopic $(<1-2 \mathrm{~mm}$ diameter $)$ clusters of haemosiderin-laden macrophages close to small vessels with impaired integrity of the blood- brain barrier. CMBs were initially considered to be clinically silent, but multiple hospitalbased and population-based MRI studies have demonstrated that they are consistently associated with not only cerebrovascular disease, but also neurocognitive disorders, including AD and subcortical vascular dementia.

Early evidence that CMBs are associated with cognitive impairment came from a small hospital-based cross-sectional case-control study, which showed that executive dysfunction in 
patients with stroke was more common among patients with CMBs than in patients without ${ }^{3}$. This observation was supported by a subsequent study of a healthy population, which demonstrated that global cognitive dysfunction measured with the Mini-Mental State Examination (MMSE; particularly the "attention and calculation" score) was independently associated with both the presence and number of $\mathrm{CMBs}^{4}$.

CMBs are of major interest because their topographical distribution is related to the likely underlying pathology (FIG. 1). Indirect evidence suggests that arteriolosclerosis (also known as hypertensive arteriopathy or deep perforator arteriopathy) affects deep basal ganglia, the brainstem and white matter perforator vessels, causing CMBs in deep (basal ganglia) or infratentorial regions and lobar regions ${ }^{5}$, whereas $\mathrm{CMBs,}$, with a strictly lobar distribution are highly specific for pathology-proven $\mathrm{CAA}^{6}$ in people with European ancestry and who have intracerebral haemorrhage, though this pattern might not be so specific in Asian people or healthy non-hospital (community) populations. Interest has, therefore, focused on whether different CMB patterns (reflecting different arteriopathies) are associated with different impacts on cognitive function, but studies have produced inconsistent results.

In the ongoing I-Lan Longitudinal Aging Study (ILAS) of randomly selected community-dwelling individuals aged $>50$ years, Chung et al. ${ }^{1}$ investigated the associations between the presence and locations of CMBs and function in a variety of cognitive domains. The study included 959 healthy individuals with a mean age of 62.5 years (SD 8.6 years), $44.3 \%$ of whom were male. CMBs were assessed with 3T 3D susceptibility imaging, and were found in 14.2\% of participants. The group with CMBs were subdivided into two groups: those with deep or infratentorial CMBs (with or without lobar CMBs) and those with strictly lobar CMBs. Global 
cognitive function was assessed with the MMSE; specific tests assessed verbal memory, language ability, visuospatial executive function and verbal executive function.

Multivariate linear logistic regression analyses, adjusted for potential confounders, were used to test whether whole-brain, deep or infratentorial, or strictly lobar CMBs (each tested separately) were associated with cognition. The results showed that the presence of CMBs was associated with global cognitive dysfunction, but that strictly lobar CMBs correlated with deficits in global cognitive and visuospatial executive functions, whereas deep or infratentorial CMBs were not associated with cognitive dysfunction. Previously, an association of cognitive impairment with strictly lobar CMBs had mainly been observed in Western cohorts, whereas in Asian populations, the association was stronger with deep or infratentorial CMBs; we suggested that these findings might have been influenced by the dominant patterns of CMBs that occur in each population - mainly strictly lobar CMBs in Western populations, and mainly deep or infratentorial CMBs in Asian populations ${ }^{5}$. The ILAS study suggests that strictly lobar CMBs could be associated with cognitive impairments, even in Asian populations ${ }^{1}$.

This study thus provides additional insight into the unclear relationship between CMBs and cognitive dysfunction. The CMBs themselves might have direct causal effects by disrupting structural or functional white matter connectivity, or by reducing cortical-subcortical connections or cortical neuronal density and function. Alternatively, CMBs might be an indirect marker for impaired structure or function of small vessels due to either CAA, deep perforator arteriopathy (arteriolosclerosis), or a combination of both ${ }^{7}$. Another key implication of the study by Chung et $a l .{ }^{1}$ is that strictly lobar CMBs might be an early (or even pre-clinical) marker of microvascular and neurodegenerative processes that underlie the pathogenesis of cognitive impairment. 
The exact mechanisms that underlie the relationship between strictly lobar CMBs and cognitive decline remain to be determined. Strictly lobar CMBs could be linked to early cognitive impairment, including preclinical $\mathrm{AD}$, through vascular amyloid- $\beta$ ( $\mathrm{A} \beta$ ) damaging the neurovascular unit causing, hypoperfusion from stenosis, impaired vascular autoregulation or reactivity, or even small areas of microinfarction ${ }^{8}$. Indeed, strictly lobar CMBs might be the "tip of iceberg" of CAA-related dysfunction in widespread cortical small vessels. On the other hand, arteriolosclerotic or CAA-related small vessel dysfunction, both of which can cause lobar CMBs, could diminish the pulsatility of arterioles, blocking bulk flow and impairing perivascular drainage and amyloid clearance from the brain.

Regardless of the underlying mechanisms, prospective evidence from another study supports the hypothesis that lobar CMBs increase the risk of cognitive decline; this study involved 3,257 non-demented healthy participants from Rotterdam, Netherlands, who were monitored for nearly 5 years ${ }^{9}$. Lobar CMBs were associated with a decline in executive function, information processing and memory function.

Identification of early processes that underlie cognitive impairment is critical to enable the design of effective treatments. Indeed, effective selection of patients in the early phase of disease by using amyloid ligand PET was probably what led to positive results in the first successful trial of immunotherapy in $\mathrm{AD}$, in which the antibody aducanumab reduced $\mathrm{A} \beta$ plaques and slowed cognitive decline ${ }^{10}$. As this study demonstrates, molecular PET imaging with ligands such as florbetapir or ${ }^{11} \mathrm{C}$-Pittsburgh compound $\mathrm{B}$ is a promising noninvasive examination for the detection of early CAA or AD, but is costly and not widely available. The ILAS study suggests that strictly lobar CMBs are relevant to early cognitive impairment, and might, therefore, offer 
Yakushiji Y \& Werring D.J.

another way to detect vascular-related amyloid deposition at an early stage.

Whether strictly lobar CMBs do indeed reflect the development of early CAA or AD in healthy cohorts could be investigated further by looking at correlations of these CMBs with cerebral atrophy measurements or amyloid deposition detected with PET. Studies that compare distributions of CMBs in people from different geographical populations and ethnicities will also be of major interest, as the spectrum of SVD that is relevant to cognition might vary worldwide. For the moment, however, this study emphasizes that CMB-related SVD, especially related to strictly lobar CMBs, plays a critical role in the earliest stages of cognitive impairment, with potential relevance to patient selection and the design of effective treatments for cognitive impairment associated with increased SVD and cerebral amyloid (including CAA or AD).

\section{Acknowledgements}

The authors acknowledge funding support for work described in this article from a Grant-in-Aid for Scientific Research (C), JSPS KAKENHI (Grant Number 15k10364), and at UCLH/UCL, 
who received a proportion of funding from the Department of Health's NIHR Biomedical Research Centres funding scheme.

\section{Competing interests}

Y.Y has received research support from Novartis Pharma K.K. D.J.W. declares honoraria from Bayer, and was UK chief investigator for a randomized controlled trial of ponezumab in cerebral amyloid angiopathy, sponsored by Pfizer.

\section{References}

1 Chung, C. P. et al. Strictly lobar cerebral microbleeds are associated with cognitive 
impairment. Stroke 47, 2497-2502 (2016).

2 Wardlaw, J. M. et al. Neuroimaging standards for research into small vessel disease and its contribution to ageing and neurodegeneration. Lancet Neurol. 12, 822-838 (2013).

3 Werring, D. J. et al. Cognitive dysfunction in patients with cerebral microbleeds on T2*weighted gradient-echo MRI. Brain 127, 2265-2275 (2004).

4 Yakushiji, Y. et al. Brain microbleeds and global cognitive function in adults without neurological disorder. Stroke 39, 3323-3328 (2008).

5 Yakushiji, Y. et al. Distributional impact of brain microbleeds on global cognitive function in adults without neurological disorder. Stroke 43, 1800-1805 (2012).

6 Knudsen, K. A., Rosand, J., Karluk, D. \& Greenberg, S. M. Clinical diagnosis of cerebral amyloid angiopathy: validation of the Boston criteria. Neurology 56, 537-539 (2001).

7 Kim, Y. J. et al. Synergistic effects of longitudinal amyloid and vascular changes on lobar microbleeds. Neurology, http://dx.doi.org/10.1212/WNL.0000000000003220 (2016).

8 Cordonnier, C. \& van der Flier, W. M. Brain microbleeds and Alzheimer's disease: innocent observation or key player? Brain 134, 335-344 (2011).

9 Akoudad, S. et al. Association of cerebral microbleeds with cognitive decline and dementia. JAMA Neurol. 73, 934-943 (2016).

10 Sevigny, J. et al. The antibody aducanumab reduces $A \beta$ plaques in Alzheimer's disease. Nature 537, 50-56 (2016).

\section{Figure legends}

Figure 1 | Anatomical location of cerebral microbleeds (CMBs) in cerebral amyloid angiopathy (CAA) and deep perforator arteriopathy (arteriolosclerosis). Typical locations of CMBs are shown as dark red spots in the brain parenchyma. a CAA. Amyloid- $\beta$ peptide 
Yakushiji Y \& Werring D.J.

accumulates in the walls of small and medium arteries and superficial perforating arterioles (solid line square) strictly located in the leptomeninges and the cerebral cortex. $\mathrm{b} \mid$ Deep perforator arteriopathy (arteriolosclerosis). The deep basal ganglia, brainstem and white matter (superficial) perforating arteries are affected (dashed square), causing CMBs in deep (basal ganglia) or infratentorial regions, as well as lobar regions. 